\title{
Dissolving star cluster candidates
}

\author{
E. Bica, B. X. Santiago, C. M. Dutra, H. Dottori, M. R. de Oliveira, and D. Pavani \\ Universidade Federal do Rio Grande do Sul, IF, CP 15051, Porto Alegre 91501-970, RS, Brazil \\ Received 13 July 2000 / Accepted 10 November 2000

\begin{abstract}
We present a list of 34 neglected entries from star cluster catalogues located at relatively high galactic latitudes $\left(|b|>15^{\circ}\right)$ which appear to be candidate late stages of star cluster dynamical evolution. Although underpopulated with respect to usual open clusters, they still present a high number density contrast as compared to the galactic field. This was verified by means of (i) predicted model counts from different galactic subsystems in the same direction, and (ii) Guide Star Catalog equal solid angle counts for the object and surrounding fields. This suggests that the objects are physical systems, possibly star clusters in the process of disruption or their fossil remains. The sample will be useful for followup studies in view of verifying their physical nature.
\end{abstract}

Key words. galaxy: open clusters and associations: general

\section{Introduction}

Star Clusters are known to dynamically evolve and stellar depletion effects eventually lead to the cluster dissolution. Fundamental questions are: (i) where are the clusters in process of dissolution? (ii) if fossils are left, can any remnant be detected? The present study aims at showing that several candidates for these effects occur in star cluster catalogues themselves.

Several poorly populated objects at relatively high galactic latitudes (from the open cluster perspective $|b|>$ $15^{\circ}$ ) are included in open cluster catalogues (Alter et al. 1970; Lyngå 1987). Other objects were reported as clusters in early studies (e.g. New General Catalogue and Index Catalogue), or in modern ones like the ESO (B) Atlas Survey Catalogue (Lauberts 1982).

Depletion of Main Sequence (MS) stars has been detected or evidence of it has been found in luminosity functions and Colour-Magnitude Diagrams (CMDs) of some Palomar or Palomar-like globular clusters such as E3 (McClure et al. 1985), ESO 452-SC11 (Bica et al. 1999) and NGC 6717 (Palomar 9) (Ortolani et al. 1999). Such evolved dynamical stages of low mass globular clusters are still associated to relatively well populated star clusters, but one may wonder what subsequent stages would look like, probably an underpopulated fossil core containing some double and multiple stars.

Among open clusters low MS depletion has been found e.g. in the intermediate age $(\approx 3 \mathrm{Gyr})$ cluster NGC 3680 (Anthony-Twarog et al. 1991). The dissolution

Send offprint requests to: C. M. Dutra,

e-mail: dutra@if.ufrgs.br of open clusters has been studied by Wielen (1971) and de la Fuente Marcos (1998). Updated data suggest that most open clusters dissolve in about $100 \mathrm{Myr}$ and this will probably not change much as one includes fainter clusters (Ahumada et al. 2000). Intermediate age open clusters are certainly survivors of initially massive clusters (Friel 1995), and their updated age histogram containing more than 100 entries (Dutra \& Bica 2000) suggests a dissolution timescale of about $1 \mathrm{Gyr}$. Also, $N$-body simulations of star clusters in an external potential have shown typical dissolution times in the range 500-2500 Myr (Terlevich 1987; McMillan \& Hut 1994; de la Fuente Marcos 1997; Portegies Zwart et al. 2000). Mass segregation is expected to occur in cluster cores during one relaxation time, according to $N$-body simulations (Terlevich 1987; Portegies Zwart et al. 2000). An important effect of mass segregation is the depletion of low mass MS stars by means of evaporation due to the tidal field of the Galaxy and encounters with binary stars. This would imply that clusters which are close to disruption have a core rich in compact and giant stars (Takahashi \& Portegies Zwart 2000).

Recently, evidence of an open cluster remnant has been discussed by Bassino et al. (2000). They studied the relatively high latitude concentration of stars M 73 (NGC 6994) and derived an age of 2-3 Gyr from CMDs. They found a significant number density contrast with respect to the galactic field CMD predicted by count models in the area. Carraro (2000) does not favour the object as an open cluster or as a remnant. At any rate, if M 73 is a physical system the open cluster classification is certainly not adequate. Let us then suggest the acronym POCR Possible Open Cluster Remnant. As an ongoing study of 
this neglected class of interesting objects and in view of future CMDs to determine parameters such as reddening, age and distance, we present a list of candidates. We discuss their possible physical nature by checking whether they present a significant number density contrast with respect to their fields. In Sect. 2 we present the sample. In Sect. 3 we analyze the significance of the excesses of stars by means of (i) equal solid angle counts in the object and field areas, and (ii) galactic model counts. In Sect. 4 we discuss the results. Finally, in Sect. 5 we present the concluding remarks.

\section{The sample}

In trying to identify fossil cluster remains we browsed relatively high galactic latitude $\left(|b|>15^{\circ}\right)$ zones by means of 1st and 2nd generation Digitized Sky Survey Images and Guide Star Catalog maps, searching for poorly populated objects described as star clusters in open cluster catalogues (Alter et al. 1970; Lyngå 1987; Lauberts 1982), and also neglected NGC or IC entries originally described as clusters. Although cluster remnants must be common at low galactic latitudes we chose $|b|>15^{\circ}$ because of lower field contamination, also avoiding young disk and dust distribution details which affect models of low latitude zones (Sect. 3).

Table 1 lists the 34 POCRs and two comparison clusters, by columns: (1) object designation, (2) and (3) galactic coordinates, (4) and (5) J2000 equatorial coordinates, (6) and (7) the major and minor diameters, and finally (8) $E(B-V)_{\text {FIR }}$ reddening values. We measured coordinates of the POCR centers and diameters on Digitized Sky Survey images. The list is not intended to be extensive or complete, reflecting only the most interesting objects found in the present search. We obtained the $E(B-V)_{\mathrm{FIR}}$ reddening values from Schlegel et al. (1998) dust emission reddening maps. From the study of star cluster directions Dutra \& Bica (2000) called attention to the fact that Schlegel et al.'s reddening values reflect foreground and background dust contributions. Most objects in Table 1 have relatively high galactic latitudes and their reddening values are quite low, probably corresponding to foreground dust in the pathsight. The only exception is NGC 1901, which is projected onto the LMC disk and the high reddening value in Table 1 arises mainly from LMC dust (Dutra \& Bica 2000).

Some POCRs present differences between their major and minor diameters (Table 1) indicating ellipticity. Numerical simulations by Terlevich (1987) and Portegies Zwart et al. (2000) have shown that stars escape from the cluster due to the Galaxy tidal field preferentially through the lagrangian points located on the vector connecting the cluster to the Galactic centre. This effect tends to flatten the clusters in the direction perpendicular to the Galactic Plane. This might explain some cases of ellipticity, considering also projection effects. Ellipticity can also be explained by the long-term evolution of binary star clusters (de Oliveira et al. 2000).
In the following we comment on some objects. The designation NGC 1963 is often applied to an edge-on galaxy $\approx 11^{\prime}$ east of the POCR. However the New General Catalogue describes a star cluster, and the original position (available e.g. in Sulentic \& Tifft 1973) coincides with that of the POCR. Lauberts (1982) correctly identified the present object (NGC 1963 or ESO 363SC5) and the galaxy (IC 2135 or ESO 363G7). On the other hand the POCR NGC 2314 A has a neighbouring bright galaxy (NGC 2314 itself) located $\approx 5^{\prime}$ north. The New General Catalogue description clearly refers to the galaxy, but the designation NGC 2314 has been applied to the stellar concentration (Alter et al. 1970), which is also known as OCl-374. We suggest the extension "A" to the POCR designation. NGC 2664, NGC 1557 and NGC 1641 are poorly populated concentrations of stars reported as clusters in the NGC catalogue, but not included in modern open cluster catalogues. NGC 2664 is projected just a few cluster diameters away from the populous intermediate age open cluster M 67, an old disk template spatially located quite high in the plane $(z=430 \mathrm{pc}-$ see Mermilliod's (1996) WEBDA Open Cluster database for essential cluster parameters in the Web interface http://obswww. unige.ch/webda). NGC 1901 is an open cluster or stellar group (Sanduleak \& Philip 1968) that is projected to the north of the LMC bar, whereas NGC 1641 and NGC 1557 are projected onto the outer parts of the LMC. If physical stellar systems, these objects are clearly related to the Milky Way due to the brightness of their stars. The close projection of some open clusters and POCRs also leaves the possibility of future investigations, such as common origin, early cluster pairs or multiplets, fossil associations, or perhaps debris of dwarf galaxy captures.

We show in Fig. 1 the angular distribution of the 34 sample objects and the two comparison clusters. POCRs can be basically found in any direction along the plane. The number of POCRs is small but concentrations could be present in the third Quadrant, which would suggest that several objects therein had common origin. We emphasize that our survey of POCRs was based on previously catalogued objects, and it would be important to carry out a systematic search for new objects with similar characteristics in the same part of sky, as well as for $|b|<15^{\circ}$.

Figure 2 shows a 2nd generation Digitized Sky Survey (XDSS) image of the comparison cluster NGC 3680 which has low MS depletion (Anthony-Twarog et al. 1991). The WEBDA database provides a distance of $735 \mathrm{pc}$ which combined to the angular dimensions in Table 1 imply a linear diameter of $3.2 \mathrm{pc}$. The other comparison cluster NGC 1901 (Table 1) has an age of 830 Myr according to WEBDA. The distance is $415 \mathrm{pc}$ implying a linear diameter of 2.3 pc. NGC 3680 and NGC 1901 are probably evolving into POCRs. Figure 3 shows a XDSS image of ESO 425SC15 which resembles NGC 3680, but seems to be more depleted in terms of faint stars. Figure 4 shows a 1st generation image (DSS) of NGC 7772 which 
Table 1. The sample

\begin{tabular}{|c|c|c|c|c|c|c|c|c|c|c|c|c|c|}
\hline Name & $\begin{array}{c}\ell \\
\left({ }^{\circ}\right)\end{array}$ & $\begin{array}{c}b \\
\left({ }^{\circ}\right)\end{array}$ & $\begin{array}{c}\mathrm{RA}(2000) \\
\mathrm{h}: \mathrm{m}: \mathrm{ss}\end{array}$ & $\begin{array}{c}\operatorname{Dec}(2000) \\
\circ:^{\prime} \quad:^{\prime \prime}\end{array}$ & $\begin{array}{l}D \\
\left({ }^{\prime}\right)\end{array}$ & $\begin{array}{r}d \\
\left({ }^{\prime}\right)\end{array}$ & $E(B-V)_{\mathrm{FIR}}$ & $B_{\lim }$ & $\begin{array}{l}\text { Sol. Ang. } \\
\left(\operatorname{arcmin}^{2}\right)\end{array}$ & $\begin{array}{l}\text { Object } \\
\text { counts }\end{array}$ & $\begin{array}{l}\text { Model } \\
\text { counts }\end{array}$ & $\begin{array}{c}\text { GSC } \\
\text { counts }\end{array}$ & $\begin{array}{c}\text { GSC } \\
\text { std }\end{array}$ \\
\hline ESO $464 \mathrm{SC} 9$ & 15.92 & -39.43 & $20: 59: 37$ & $-29: 23: 12$ & 4 & 3 & 0.10 & 16.5 & 12.0 & $3^{*}(13)$ & 2.48 & $0.93^{*}$ & $1.17^{*}$ \\
\hline M 73, NGC 6994, Cr 426, OCl-89 & 35.71 & -33.93 & $20: 58: 55$ & $-12: 38: 03$ & 9 & 9 & 0.05 & 12.5 & 81.0 & 5 & 1.30 & 1.01 & 1.11 \\
\hline NGC 6863 & 38.27 & -17.99 & $20: 05: 07$ & $-3: 33: 20$ & 2 & 1.5 & 0.29 & 15.5 & 3.0 & 3 & 1.00 & 0.37 & 0.70 \\
\hline DoDz6, OCl-129 & 61.58 & 40.36 & $16: 45: 24$ & $38: 21: 00$ & 6 & 3.5 & 0.02 & 13.0 & 21.0 & 5 & 0.39 & 0.50 & 0.66 \\
\hline NGC7 036 & 64.54 & -21.44 & $21: 10: 02$ & $15: 31: 05$ & 4 & 3 & 0.08 & 14.5 & 12.0 & 5 & 1.48 & 1.75 & 1.37 \\
\hline NGC 7772, OCl-230 & 102.73 & -44.27 & $23: 51: 46$ & $16: 14: 49$ & 3 & 2 & 0.04 & 13.5 & 6.0 & 4 & 0.13 & 0.13 & 0.34 \\
\hline NGC 5385 & 118.19 & 40.38 & $13: 52: 27$ & $76: 10: 27$ & 8 & 6 & 0.04 & 12.0 & 48.0 & 7 & 0.39 & 0.54 & 0.76 \\
\hline $\mathrm{Cr} 21, \mathrm{OCl}-371$ & 138.73 & -33.99 & $1: 50: 11$ & $27: 04: 00$ & 9 & 7.5 & 0.07 & 11.0 & 67.5 & 7 & 0.26 & 0.34 & 0.57 \\
\hline NGC $2314 \mathrm{~A}$, OCl-374 & 139.58 & 27.35 & $7: 10: 12$ & $75: 12: 15$ & 4.5 & 3.5 & 0.04 & 15.5 & 15.75 & 6 & 2.02 & 1.57 & 1.50 \\
\hline NGC 2408 & 143.62 & 29.46 & 7:40:09 & $71: 39: 20$ & 24 & 20 & 0.02 & 11.0 & 480.0 & 13 & 2.43 & 2.61 & 1.59 \\
\hline DoDz1, OCl-287 & 158.61 & -37.43 & $2: 47: 30$ & $17: 16: 00$ & 14 & 9 & 0.10 & 11.0 & 126.0 & 5 & 0.48 & 0.56 & 0.82 \\
\hline NGC 1498 & 203.62 & -43.32 & 4:00:18 & $-12: 00: 55$ & 2.2 & 1.8 & 0.05 & 14.0 & 4.0 & 2 & 0.11 & 0.01 & 0.10 \\
\hline NGC 2664 & 214.34 & 31.31 & $8: 47: 11$ & $12: 36: 10$ & 8 & 8 & 0.02 & 11.3 & 64.0 & 3 & 0.39 & 0.53 & 0.70 \\
\hline ESO 489SC1 & 232.92 & -21.41 & $6: 04: 58$ & $-26: 44: 05$ & 11 & 11 & 0.03 & 13.2 & 121.0 & 12 & 4.89 & 4.02 & 1.90 \\
\hline ESO $425 \mathrm{SC} 6$ & 235.39 & -22.28 & $6: 04: 50$ & $-29: 10: 59$ & 6 & 6 & 0.03 & 15.0 & 36.0 & 13 & 4.67 & 4.74 & 2.23 \\
\hline ESO $425 \mathrm{SC} 15$ & 236.37 & -20.35 & $6: 14: 35$ & $-29: 22: 30$ & 6 & 6 & 0.04 & 14.5 & 36.0 & 10 & 3.80 & 3.84 & 1.89 \\
\hline $\mathrm{ESO} 424 \mathrm{SC} 25$ & 237.72 & -26.37 & $5: 49: 49$ & $-32: 28: 20$ & 9 & 7 & 0.04 & 13.0 & 63.0 & 9 & 1.71 & 1.59 & 1.33 \\
\hline ESO $426 \mathrm{SC} 26$ & 239.62 & -16.51 & $6: 36: 18$ & $-30: 51: 30$ & 7 & 7 & 0.11 & 14.5 & 49.0 & 15 & 6.47 & 5.85 & 2.52 \\
\hline NGC 1891, ESO $362 ? 20$ & 239.69 & -32.87 & $5: 21: 25$ & $-35: 44: 29$ & 15 & 10 & 0.04 & 12.5 & 150.0 & 6 & 2.19 & 1.43 & 1.20 \\
\hline NGC 1963, ESO $363 \mathrm{SC} 5$ & 240.99 & -30.86 & $5: 32: 17$ & $-36: 23: 30$ & 13 & 13 & 0.03 & 13.0 & 169.0 & 13 & 3.81 & 3.06 & 1.90 \\
\hline ESO 437SC61 & 273.06 & 26.22 & 10:48:03 & $-29: 23: 28$ & 5 & 4 & 0.07 & 16.0 & 20.0 & $5^{*}(9)$ & 4.35 & $2.20^{*}$ & $1.38^{*}$ \\
\hline ESO 245SC9 & 273.76 & -67.48 & $1: 53: 43$ & $-45: 57: 16$ & 14 & 12 & 0.02 & 13.5 & 168.0 & 11 & 2.41 & 2.36 & 1.50 \\
\hline ESO $570 \mathrm{SC} 12$ & 274.06 & 35.92 & $11: 12: 12$ & $-21: 19: 13$ & 13 & 13 & 0.03 & 12.8 & 169.0 & 10 & 3.01 & 2.15 & 1.40 \\
\hline NGC 1252, ESO 116?11 & 274.08 & -50.83 & $3: 10: 49$ & $-57: 46: 00$ & 14 & 11 & 0.02 & 12.0 & 154.0 & 8 & 1.02 & 1.04 & 1.00 \\
\hline ESO $502 \mathrm{SC} 19$ & 276.02 & 30.68 & 11:08:07 & $-26: 43: 51$ & 3 & 3 & 0.05 & 15.0 & 9.0 & 3 & 0.87 & 0.63 & 0.75 \\
\hline NGC 1641, ESO $84 \mathrm{SC} 24$ & 277.20 & -38.31 & $4: 35: 32$ & $-65: 45: 03$ & 11 & 9 & 0.04 & 13.5 & 99.0 & 11 & 2.64 & 2.86 & 1.72 \\
\hline NGC 2348, ESO $88 \mathrm{SC} 1$ & 278.14 & -23.80 & 7:03:03 & $-67: 24: 44$ & 18 & 10 & 0.12 & 13.0 & 100.0 & 5 & 3.28 & 2.57 & 1.61 \\
\hline IC 1023, ESO $385 \mathrm{SC} 39$ & 324.95 & 22.71 & $14: 32: 25$ & $-35: 48: 13$ & 5 & 5 & 0.07 & 15.0 & 25.0 & 13 & 4.72 & 3.19 & 1.55 \\
\hline ESO $141 \mathrm{SC} 47$ & 338.95 & -26.17 & 19:18:03 & $-57: 54: 16$ & 7 & 5 & 0.06 & 14.5 & 35.0 & 11 & 3.93 & 2.62 & 1.59 \\
\hline \multirow[t]{2}{*}{ ESO $282 \mathrm{SC} 26$} & 355.01 & -21.89 & $19: 13: 52$ & $-42: 38: 58$ & 15 & 13 & 0.08 & 12.5 & 195.0 & 12 & 6.26 & 3.43 & 1.70 \\
\hline & & & Comparison & Clusters & & & & & & & & & \\
\hline NGC 3680, Mel-106 & 286.76 & 16.92 & $11: 25: 38$ & $-43: 14: 30$ & 15 & 15 & 0.09 & $10.9-12.5$ & 225.0 & 20 & 6.14 & 3.55 & 2.14 \\
\hline NGC 1901, Bok 1, OCl-791.1 & 279.03 & -33.60 & $5: 18: 11$ & $-68: 27: 00$ & 19 & 19 & 0.36 & 10.5 & 361.0 & 9 & 0.91 & 2.63 & 1.88 \\
\hline
\end{tabular}

* GSC not deep enough to reach assumed $B_{\text {lim }}$. Number in parenthesis comes from DSS visual counts.

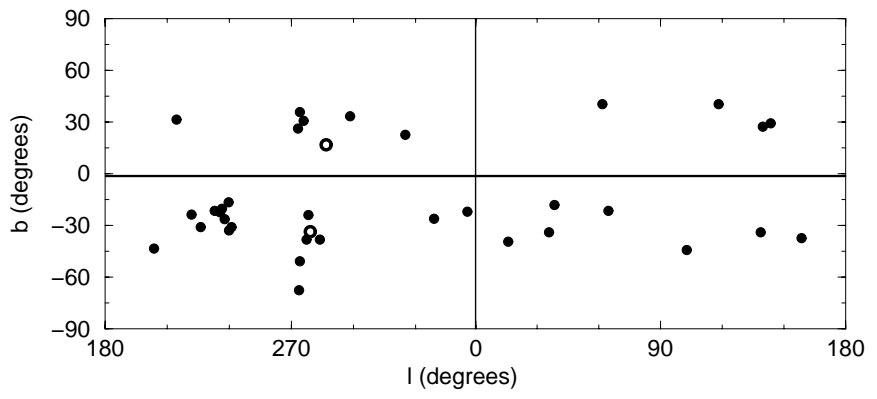

Fig. 1. Angular distribution of the 34 POCRs (filled circles) together with the comparison clusters NGC 3680 and NGC 1901 (open circles). Galactic Plane and Minor Axis are indicated by solid lines

is a more compact POCR. Finally, Fig. 5 shows a XDSS image of NGC 1252 which is a POCR with a rather loose morphology. The New General Catalogue describes NGC 1252 as a star cluster with 18-20 stars. This descrip- tion is compatible with the only concentration of stars in the area, which is the present POCR with dimensions $14^{\prime} \times$ $11^{\prime}$ (Table 1). Bouchet \& Thé (1983) carried out photometry in a region with diameter $\approx 1^{\circ}$ centred on the bright carbon star TW Horologii. The POCR is located at the edge of Bouchet \& Thé's large region. The only star in the POCR area considered to be member of the cluster as interpreted by Bouchet \& Thé is BT1. Recently Baumgardt (1998) discussed 12 bright stars in Bouchet \& Thé's large region based on the ACT and Hipparcos catalogues and concluded that those stars do not make up a cluster. The present POCR which is to be identified with NGC 1252 has not yet been explored.

\section{Number density contrast analysis}

Guide Star Catalog 1.1 (hereafter, GSC) maps and/or DSS/XDSS images clearly show that the present objects stand out from their surrounding fields. In the following 


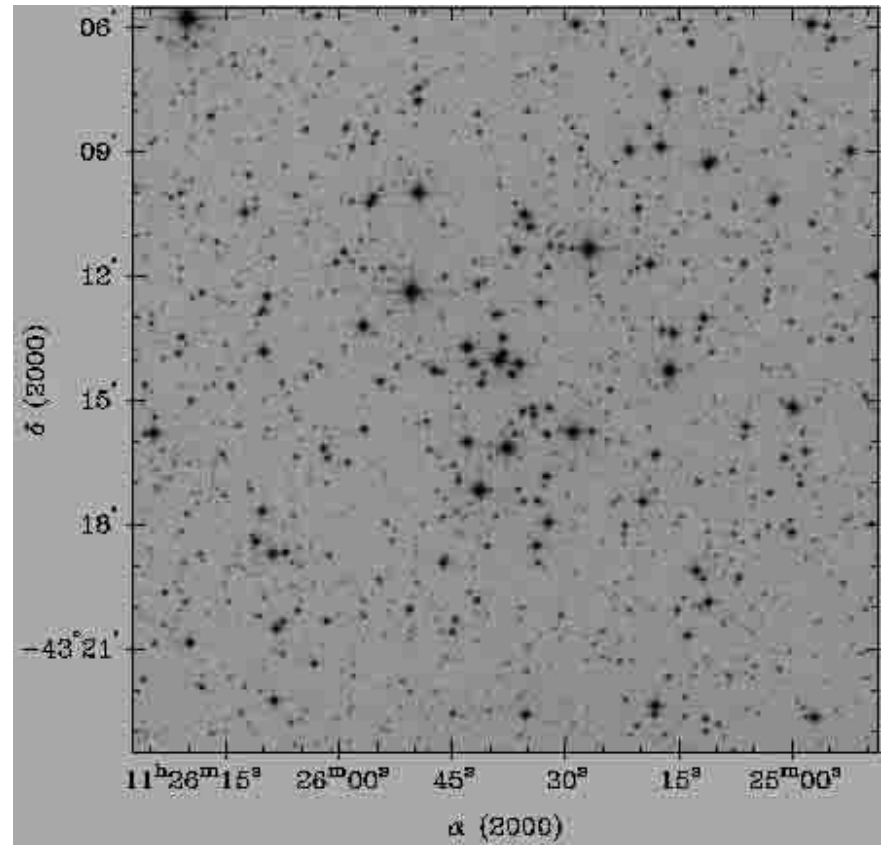

Fig. 2. $18 \times 18 \operatorname{arcmin}^{2}$ XDSS image of the depleted low Main Sequence open cluster NGC 3680

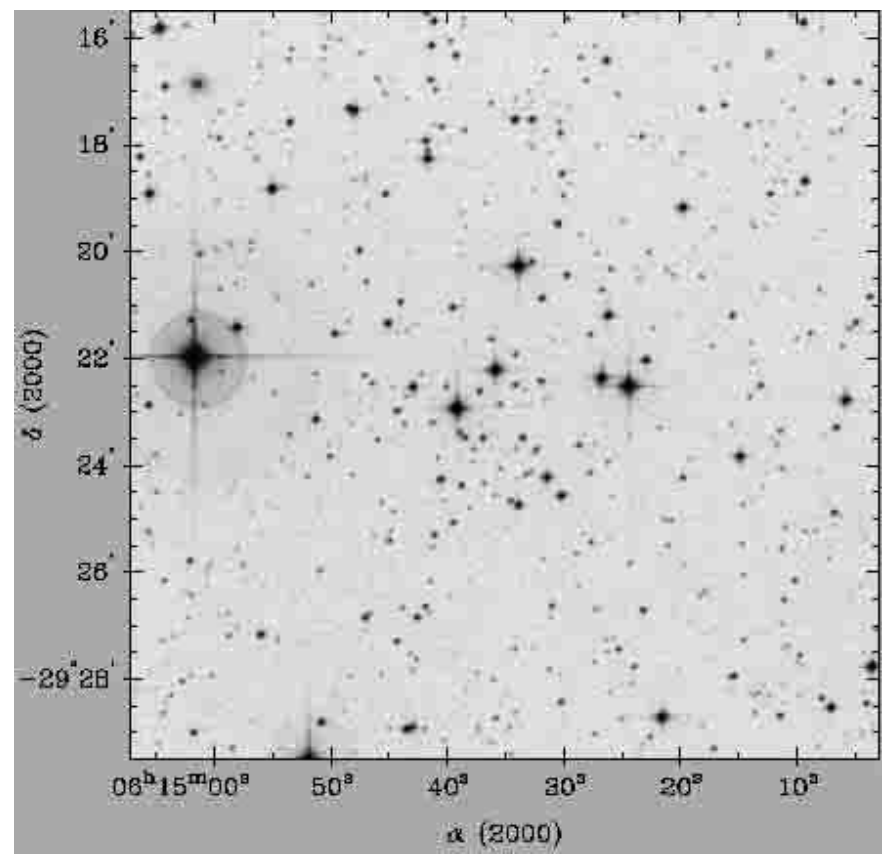

Fig. 3. $14 \times 14 \operatorname{arcmin}^{2}$ XDSS image of the POCR ESO 425SC15

we assess the statistical significance of the excess projected number density of stars represented by the POCRs that allowed previous authors to identify them on photographic plates. We use two independent methods to analyze the number density contrast: (i) comparing star counts from DSS images in the cluster region up to a limiting magnitude at which the cluster prevails over the neighbouring field, with theoretical star counts based on a Galactic Structure Model predicted up to the same magnitude; (ii) the same cluster counts are compared to counts in neigh-

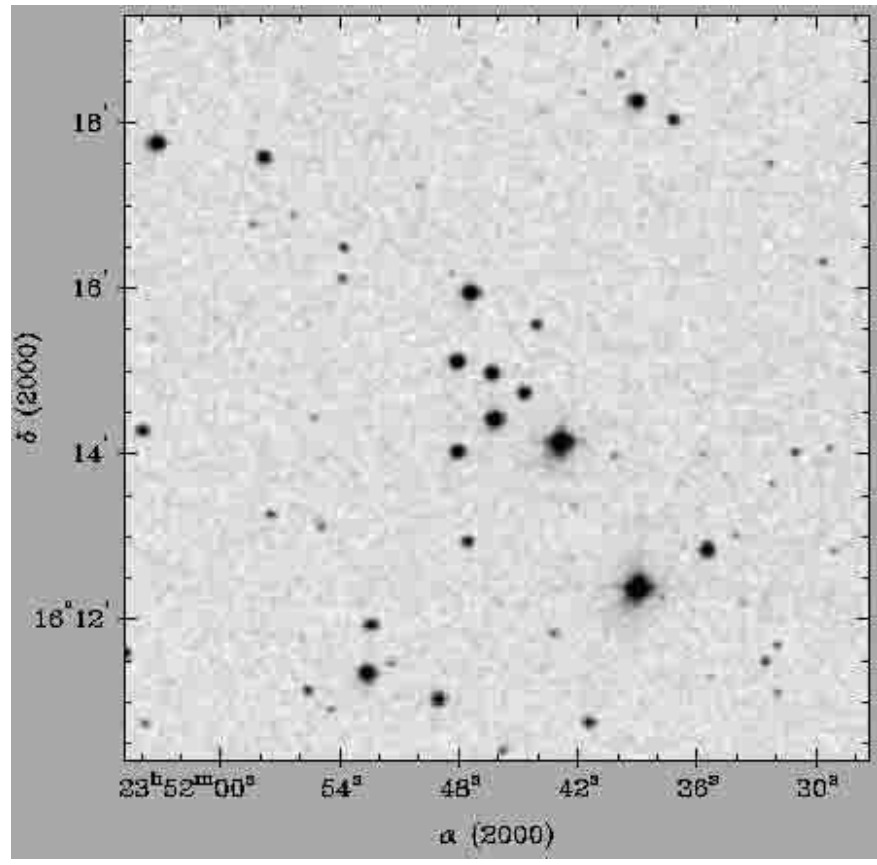

Fig. 4. $9 \times 9 \operatorname{arcmin}^{2}$ DSS image of the POCR NGC 7772

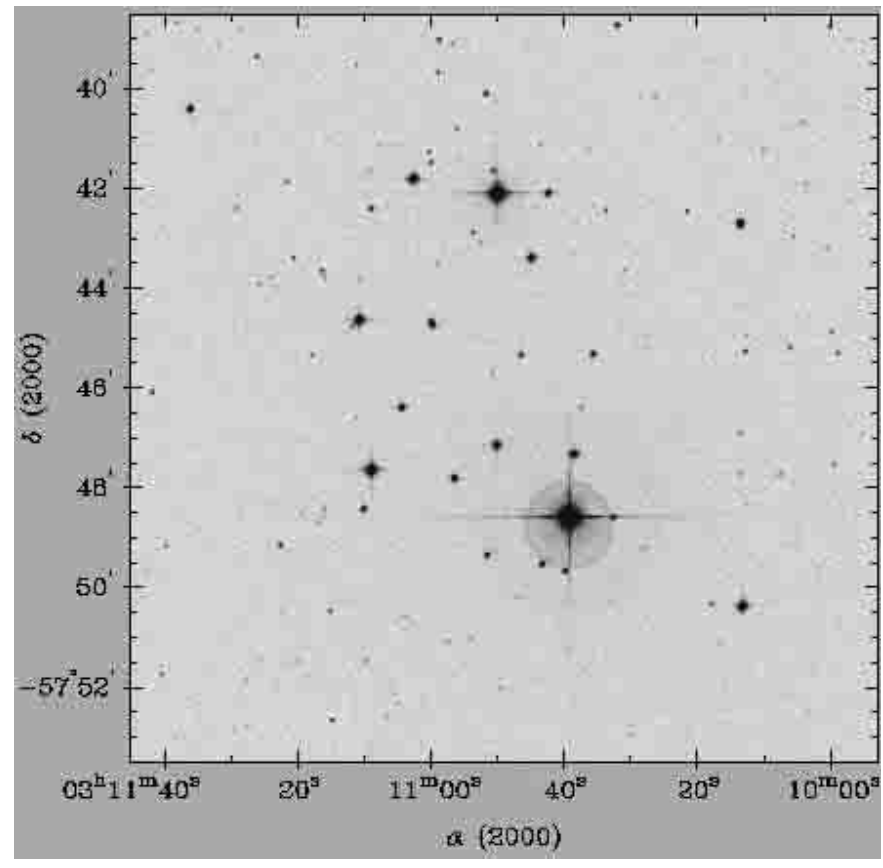

Fig. 5. $15 \times 15 \operatorname{arcmin}^{2}$ XDSS image of the POCR NGC 1252

bouring GSC fields with solid angle equal to the cluster's. The magnitude range responsible for the excess stars was visually defined for each POCR using DSS and XDSS images and the GSC positions and magnitudes.

The adopted Galactic Model has been previously described in more detail (Santiago et al. 1996; Reid \& Majewski 1993). It includes 3 structural components: a thin disk, a thick disc and a spheroid. Given the typical magnitude limit for the cluster stars $(V \approx 15)$, our relevant star counts are almost entirely dominated by the thin disk. Its stellar density profile is described by a double 


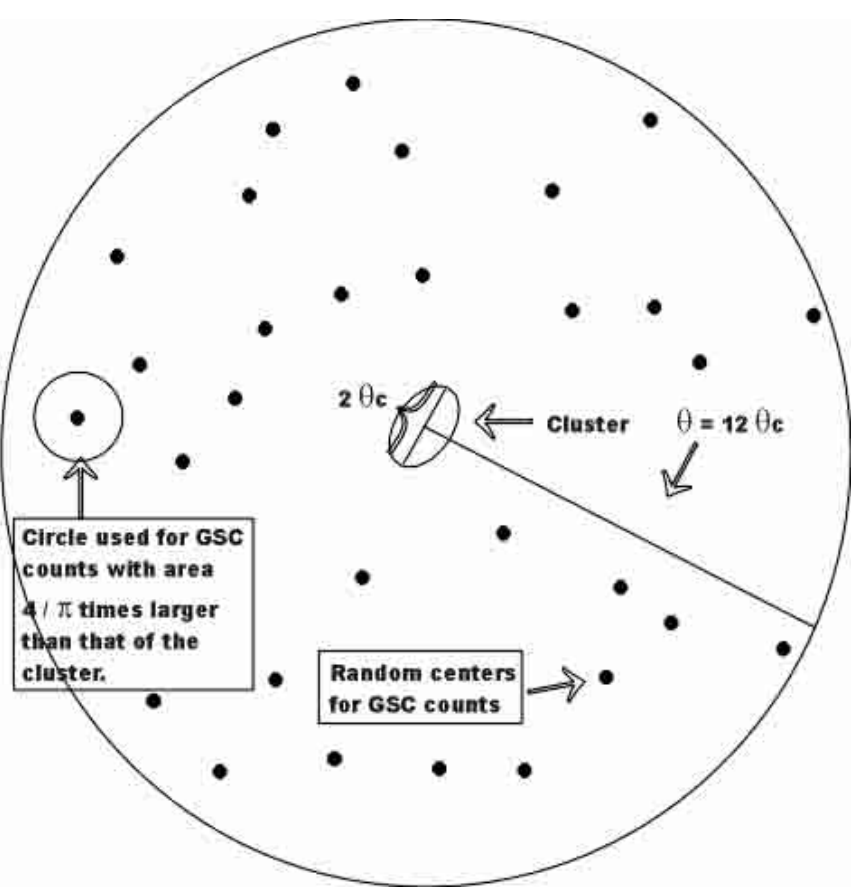

Fig. 6. Schematic representation of the procedure employed to count stars in the object and surrounding field samplings

exponential, one along the plane of the disk and the other perpendicular to it. As for the luminosity function, it is very well constrained for disc stars with $M_{V} \approx 10$, which will largely dominate the field star number counts in the DSS images and GSC catalogue (Wielen et al. 1983; Bessel \& Stringfellow 1993). We therefore anticipate no model uncertainties propagating into the predicted number counts of field stars.

The GSC fields were extracted using the task REGIONS in the STSDAS package inside IRAF. With the positions and magnitudes of all GSC stars within $12 \theta_{\text {clus }}$, where $\theta_{\text {clus }}$ is the apparent major diameter of each POCR, defined from inspection of the DSS images, we randomly selected 100 centers within this region and counted the number of GSC stars found within the same solid angle and magnitude range defined for the POCR. The procedure is illustrated by Fig. 6. The model and GSC counts were taken from a solid angle $\omega=D d$, which corresponds to a rectangle that encompasses the cluster ellipse, therefore being larger than that of the cluster by a factor $4 / \pi$. This conservative procedure of estimating expected field star counts is an attempt to compensate for uncertainties in the defined cluster boundaries.

The results of both experiments are given in Cols. (9) through (14) of Table 1 . We show the limiting magnitude (in two cases bright field stars also occurred so that a bright limit is also shown), solid angle and number of stars found for the POCR. The next column lists the expected number of stars from the model in the same region. Finally, we list the average and dispersion of the GSC star counts in randomly placed areas in the cluster's neighbourhood.
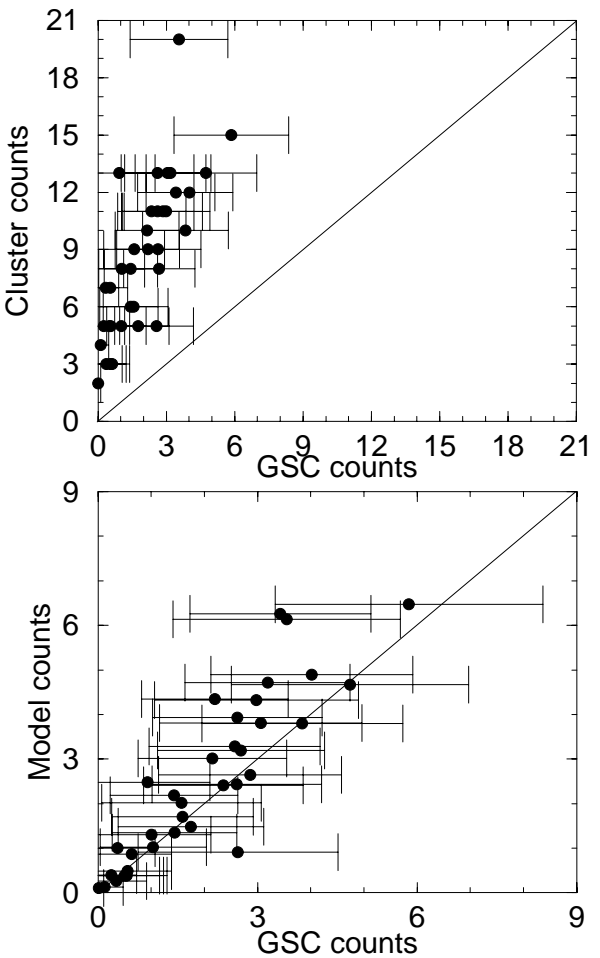

Fig. 7. Count comparisons with those from the Guide Star Catalogue. Upper panel: counts in the object solid angle. Lower panel: Galactic Model counts. Error bars indicate $1 \sigma$ deviations from average GSC counts

Most entries clearly show an excess of observed stars relative to both model expected values and GSC counts. This is clearly seen in the upper panel of Fig. 7, where cluster counts are plotted against the average GSC counts. Exceptions are ESO 464SC9, ESO 437SC61 and NGC 2348, but in the former two the GSC is not deep enough to reach the assumed $B_{\text {lim }}$ (Table 1 ). For all other POCRs, the observed counts well exceed the dispersion of GSC counts relative to the mean value. The high concentration of stars in M 73 (NGC 6994) occurs in a $2^{\prime} \times 2^{\prime}$ region (Bassino et al. 2000; Carraro 2000). However the diameter in Table 1 of $9^{\prime} \times 9^{\prime}$ corresponds to a halo region with probable members (Bassino et al. 2000). This larger area provides a conservative estimate of the number density contrast, which is significant (Table 1 ). The distance of $620 \mathrm{pc}$ (Bassino et al. 2000) implies linear diameters of $1.2 \mathrm{pc}$ and $0.4 \mathrm{pc}$ for the halo and core regions respectively. These estimates can be compared with those of the dynamically evolved clusters NGC 3680 and NGC 1901 (Sect. 2), resulting smaller for the POCR.

The two estimates of background counts, model and GSC, agree quite closely, as shown in the lower panel of Fig. 7. The reason why model counts lie, in average, slightly above the GSC ones is likely caused by incompleteness effects affecting the latter. This consistency in the two independent estimated numbers of field stars and the high number density contrasts support the possibility of the POCRs being real physical systems. 


\section{Discussion}

The number density contrast of stars in the objects with respect to the field resulted significant (Sect. 3), revealing a real concentration, and this is the first step towards establishing a possible physical nature. The construction of the spatial distribution will require CMDs, but their high latitude locations give some hints on their origin as galactic subsystems. Note that M 73's age and position (Bassino et al. 2000) place it in the old disk. The available similar properties and angular distributions suggest the old disk as the likely origin for most POCRs in Table 1. It is not unexpected from the high latitudes that a globular cluster remnant be present. Spectroscopic and velocity studies might readily reveal halo characteristics. It is not excluded either that the sample includes a thick disk (Gilmore \& Reid 1983; Yoshii et al. 1987) cluster remnant. The scale height attributed to the thick disk is about $1 \mathrm{kpc}$ (Kerber et al. 2000), and it should be very old, near the upper age limit of the old disk. In this scenario the thick disk would be a direct halo/disk transition as gas settled into the disk forming stars. Alternatively, the thick disk could be a thin disk population heated by some internal or external mechanism such as a bar or by dwarf galaxy captures throughout the Galaxy history (Freeman 1992). In the latter scenario clusters with $t \gtrsim 500$ Myr would be expected at relatively large heights. Some orbital debris might be identified by means of high latitude clumps of star clusters and/or their fossils, which also increases the importance of Table 1 objects.

The evolutionary stage and duration of the POCR phase in the lifetime of a star cluster can be inferred from available numerical models. Assuming that a cluster becomes significantly depopulated with a POCR appearance by losing $2 / 3$ of its stars, simulations of clusters of initial mass $1000 M_{\odot}$ with various mass spectra and concentration degrees (Terlevich 1987) show that the depopulated phase lasts $\Delta t \approx 200 \mathrm{Myr}$ for a total lifetime of $\approx 1 \mathrm{Gyr}$. The timescale from the depopulated phase until dissolution would be about 400 Myr. Portegies Zwart et al. (2000) computed models for $1500 M_{\odot}$ considering a range of galactocentric distances. For a distance $R_{\mathrm{GC}} \approx 12 \mathrm{kpc}$ the cluster is more stable and the POCR phase could last much longer than above. For $R_{\mathrm{GC}} \approx 6 \mathrm{kpc}$ the timescales are comparable to those of the $1000 M_{\odot}$ clusters computed by Terlevich (1987). For less massive model clusters (Terlevich 1987) the POCR phase would be typically $1 / 3$ to $1 / 4$ of the cluster lifetime. Considering a catalogued population of open clusters of $\approx 1000$ (Lyngå 1987; Alter et al. 1970) as many as 500 POCRs would be expected in all galactic latitudes. Considering the known sample of intermediate age open clusters (Dutra \& Bica 2000) we expect $\approx 50$ POCRs of such ages. Clearly, a complete census of POCRs requires additional surveys, in particular for lower galactic latitudes. Detailed observations of each object are necessary to assess their physical properties and as a consequence to shed more light on the last stages of star cluster dynamical evolution.

\section{Concluding remarks}

We presented a list of 34 neglected entries from star cluster catalogues located at relatively high galactic latitudes $\left(|b|>15^{\circ}\right)$ which may be late stages of cluster dynamical evolution. Although underpopulated with respect to usual open clusters, we showed that they still present a high number density contrast with respect to the galactic field, as verified by means of (i) predicted model counts from different galactic subsystems in the same direction, and (ii) Guide Star Catalog equal solid angle counts for the object and surrounding fields. The sample will be useful for followup studies, aimed at verifying their physical nature. Photometry and spectroscopy are required to determine fundamental parameters such as reddening, distance, age, radial velocities, membership and chemical abundances. Some of these objects might be clusters in the process of disruption or their fossil remnants. The dynamical state of the physical objects in the present sample may be inferred from comparisons of the cluster remnant and surrounding field luminosity functions, searching for depletion effects.

An important population of possible open cluster remnants is likely to exist. They may survive significant amounts of time as depopulated systems before dissolution. Simple arguments based on available numerical models and catalogued open clusters suggest that several hundreds can be expected. Systematic surveys to find new candidates and numerical models to explore in detail the evolved dynamical stages are encouraged for a better understanding of this so far overlooked object class.

Acknowledgements. We use data from The Guide Star Catalog 1.1 and Digitized Sky Survey which were produced at the Space Telescope Science Institute under U.S. Government grants NAS5-26555 and NAG W-2166, respectively. These data are based on photographic data obtained using the Oschin Schmidt Telescope on Palomar Mountain and the UK Schmidt Telescope. We thank the referee Dr. Simon Portegies Zwart, for interesting remarks. We acknowledge support from the Brazilian institution CNPq.

\section{References}

Ahumada, A. V., Clariá, J. J., Bica, E., \& Piatti, A. E. 2000, A\&AS, 141, 79

Alter, G., Ruprecht, J., \& Vańysek, J. 1970, Catalogue of Star Clusters and Associations, ed. G. Alter, B. Balázs, \& J. Ruprecht (Akademiai Kiado, Budapest)

Anthony-Twarog, B. J., Heim, E. A., Twarog, B. A., \& Caldwell, N. 1991, AJ, 102, 1056

Bassino, L. P., Waldhausen, S., \& Martínez, R. E. 2000, A\&A, 355,138

Baumgardt, H. 1998, A\&A, 340, 402

Bessel, M. S., \& Stringfellow, G. S. 1993, ARA\&A, 31, 433

Bica, E., Ortolani, S., \& Barbuy, B. 1999, A\&AS, 136, 363

Bouchet, R., \& Thé, P. S. 1983, PASP, 95, 474

Carraro, G. 2000, A\&A, 357, 145

de la Fuente Marcos, R. 1997, A\&A, 322, 764

de la Fuente Marcos, R. 1998, A\&A, 333, L27

de Oliveira, M. R., Bica, E., \& Dottori, H. 2000, MNRAS, 311 589

Dutra, C. M., \& Bica, E. 2000, A\&A, 359, 347 
Freeman, K. C. 1992, The Stellar Population of Galaxies, ed.

B. Barbuy, \& A. Renzini (Kluwer: Dordrecht), 65

Friel, E. D. 1995, ARA\&A, 33, 381

Gilmore, G., \& Reid, N. 1983, MNRAS, 202, 1025

Kerber, L. O., Javiel, S., \& Santiago, B. X. 2000, A\&A, in press

Lauberts, A. 1982, The ESO/Uppsala Survey of the ESO

(B) Atlas, European Southern Observatory, Garching bei München

Lyngå, G. 1987, Catalog of Open Star Cluster Data, Strasbourg CDS

McClure, R. D., Hesser, J. E., Stetson, P. B., \& Stryker, L. L. 1985, PASP, 97, 665

McMillan, S., \& Hut, P. 1994, ApJ, 427, 793

Mermilliod, J. C. 1996, in The origins, evolution, and destinies of binary stars in clusters, ASP Conf. Ser., 90, 475

Ortolani, S., Barbuy, B., \& Bica, E. 1999, A\&AS, 136, 237

Portegies Zwart, S. F., McMillan, S. L. W., Hut, P., \& Makino, J. 2000, MNRAS, submitted [astro-ph/0005248]
Reid, I. N., \& Majewski, S. R. 1993, ApJ, 409, 635

Sanduleak, N., \& Philip, A. G. D. 1968, AJ, 73, 566

Santiago, B. X., Gilmore, G., \& Elson, R. 1996, MNRAS, 281, 871

Schlegel, D. J., Finkbeiner, D. P., \& Davis, M. 1998, ApJ, 500, 525

Sulentic, J. W., \& Tiff, W. G. 1973, The Revised New General Catalogue of nonstellar astronomical objects (The University of Arizona Press, Tucson)

Terlevich, E. 1987, MNRAS, 224, 193

Takahashi, K., \& Portegies Zwart, S. F. 2000, ApJ, 535, 759

Wielen, R. 1971, A\&A, 13, 309

Wielen, R., Jahreiss, H., \& Krüger, R. 1983, in IAU Coll. 76, Nearby Stars and the Stellar Luminosity Function, ed. A. G. D. Philip, \& A. R. Upgren (L. Davis Press: Schenectady), 163

Yoshii, Y., Ishida, K., \& Stobie, R. S. 1987, AJ, 93323 